\section{PROSTATIC HYPERTROPHY.}

\section{RAMON GUITERAS, M.D.}

Professor of Genito-urinary Surgery, New York Post-Graduate Medical School; Lecturer in the Medical Department of New York University. NEW YORK.

SOME OBSERVATIONS ON THE BOTTINI OPERATION.

One of the curses of old age is hypertrophy of the prostrate. Why it occurs in some unfortunates and not in others is a question that is difficult to answer, and if it could be answered it would then be possible to learn the cause, a question which now is such a puzzling one to the most learned and observing surgeons. It would seem natural to suppose that those who had in early youth suffered from venereal troubles, or had indulged too freely in the dissipation of life, would be the ones picked out for punishment in old age, but such does not seem to be the case, and in my personal experience it seems rather an unjust reward for the steady, the forbearing and the righteous.

To describe just what this condition is would be to unfold a mystery which the most distinguished pathologists have apparently not yet fathomed. For although many, in describing its pathology, make it easy to understand, others seem to be at variance with their opinion, e. g., some pathologists say that it is always a fibromyomatous growth and never a glandular one, while others say that it is often glandular.

The cause and pathology are, then, the two great points to be considered, and it would seem that, where there are so many eminent pathologists connected with the homes for aged men, autopsies ought to disclose the latter condition and there should be unanimity of opinion, as pathology is an exact branch. It seems to me, therefore, that the inference must be drawn that the subject has been sufficiently studied and that the pathologists neglect to examine, in a routine way, this little gland tucked down between the rectum and the pubes, in the same way that they overlooked the vermiform appendix before it began to play such an important role in the surgery of the day.

To speak superficially of the pathology as it is considered today, it would seem that enlargements of the prostrate are variously classified according to their microscopic anatomy or the clinic symptoms to which they give rise. As the symptoms are chiefly due to the physical qualities of the tumor and are thus mechanic in their origin, it is manifestly more important to classify prostatic hypertrophies by their macroscopic appearance rather than by their microscopic. But at the same time it is worth mentioning that there are supposed to be three chief varieties histologically: 1, myomatous, or fibromyomatous, in which the stroma of the gland is principally involved; 2 , adenomatous, in which the glandular elements predominate; and, 3, mixed forms, in which both stroma and glandular tissues are involved.

Such specialization, according to Socin, has but little surgical interest, because in clinic diagnosis it cannot be applied to good account and thus has no influence on prognosis or treatment.

$V$ iewed clinically, prostate hypertrophies occur in three chief forms.

1. Uniform enlargement in all directions, with the general form of the gland preserved. As the symphisis pubis and the urogenital diaphragm prevent the gland from growing downward to any great degree, the

*Presented to the Section on Surgery and Anatomy, at the Fiftieth Annual Meeting of the American Medical Association, held in Columbus,
Ohio, June 6-9, 1899 . tendency of the tumor is upward and backward into the cavity of the bladder. Thus about the orifice of the urethra is formed a ring of thickenen gland tissue under the mucous membrane of the bladder. These forms of hypertrophy do not reach so great a development as others and are more mild clinically.

2. Irregular enlargement. By far the greatest number of cases belong to this variety. All parts of the gland may be enlarged, but certain portions are much larger than others, and thus many kinds of tumors are formed. The part chiefly involved is that portion of the gland just behind the beginning of the urethra. In the first form mentioned this part fades into the lateral lobes of the enlarged gland without sharp demarcation, but here it forms a tongue which overhangs the internal orifice and is termed by Mercier the "valvule prostatique." This increases to a large independent tumor which is separated from the rest of the gland by deep grooves, and thus gives rise to the well-known "middle lobe" of the prostate.

If both lateral lobes be enlarged at the same time, we have a threefold gland clearly marked, with an eggshaped tumor on each side and a triangular pyramid behind; an arrangement which gives to the urethral orifice a " $Y$ " shape, with the stem forward and the legs backward. The surfaces of the tumors may be smooth, or, more frequently, they are irregularly prominent, as if made up of a number of smaller tumors which project into the bladder. These nodules may separate and remain as free bodies under the mucous membrane of the bladder. The anterior commissure of the gland is seldom the seat of the hypertrophy, because there are but few if any glandular elements presented here.

3. The third form occurs when all the gland is normal except in one small area, and is termed the localized variety. It is a very rare form.

As the prostate hypertrophies, the orifice of the urethra becomes elevated, and the return flow of blood from the vesical veins is impeded by pressure on the prostatic plexus. This results in imperfect evacuation of the bladder, and consequetly in residual urine. Cystitis develops, as is evidenced by a frequent desire to urinate-due to irritation of the neck of the bladder from venous congestion-and the other symptoms usually present. If the hypertrophy continues, these symptoms increase or are replaced by graver ones, and we have dilatation of the bladder, hypertrophy of the muscular and fibrous coats, and the formation of diverticula; dilation of the ureters and pelvis of the kidney, congestion and catarrhal inflammation of the entire urinary tract, with an accumulation of the urinary and inflammatory products, and perhaps septic inflammation extending from the bladder to the kidney-pyelonephritis-resulting in chronic uremia and death.

'Thus we see what conditions must be relieved in order to benefit our patient. His general health should be improved; his bowels regulated, and his skin kept active by warm baths; his clothing should be warm, to prevent taking cold, and he should eat moderately and drink plenty of water. His urine should be kept in the best possible condition by the use of internal urinary antiseptics, as the benzoates, the salicylates, boric acid, etc. Oleum gaultheriae, encalyptus, and urotropin are also of great service. Locally, his bladder should be emptied by the catheter twice a day and washed out with a boric acid solution; other antiseptic solutions, as permanganate of potassium, nitrate of silver, and borolyptol, being used at intervals. When the patient has been brought into the best possible condition gen- 
erally and locally, an operative procedure can be considered, but an operation should never be performed until palliative means have failed.

Ligation of the internal iliacs, resection of the vas deferens, castration, prostatectomy, and the formation of a suprapubic fistula have all had their day and advocates, and the mass of practitioners seem now to be coming to the opinion that the operation by the Bottini galvanocaustic incisor is in most cases the quickest, the simplest and the best.

This instrument was devised by Bottini of Pavia. When he performed his first operations-18\%5-he made use of the so-called "cauterizatore prostatico," representing an instrument of the shape of a catheter of medium caliber, with a short beak, the latter carrying on a porcelain disc a platinum plate about three-fourths of an inch long. With this plate, made red-hot by the electric current, he cauterized the prostate thoroughly at different spots, if necessary repeatedly. When the eschar had been pushed off, improvement often began to set in ; at times it took thirty days before the patients could notice the effect of the interference.

Two years later he was able to publish five successful caves treated in this way. With increasing experience, he discarded the cauterizator and made use only of the second instrument, the "incisore prostatico," which remores the mechanic obstruction to the outflow of the urine at the neck of the bladder by slowly burning a groove or grooves through the same, and not by superficial destruction. This instrument has a male and female shaft, not unlike a lithotrite. The beak of the female part forms almost a right angle with its shank, and has along its concave surface a deep groove along which the male shat glides. The shank of the male arm shows a platinum knife at its distal end, about fivecighth of an inch long, which is drawn along the groove of the female arm, toward the handle, on turning an Archimedean screw at the outer end of the instrument. $A$ scale (Sc.) attached to the latter admits of exactly gauging the length of the groove to be cut. The instrument further slows the so-called cooling apparatus, which was added to it in 1882. Freudenburg has made a modification of this instrument, which is the one usually used in Germany and this country. It differs principally in having a larger and better-cooled handle, and the water-pipes at a more acute angle to it. It is also a heavier instrument.

This operation has been performed about two hundred times, but I have only found about one hundred wellreported cases, exclusive of my own.

Bottini has performed this operation 80 times, but we have found only 23 cases sufficiently well reported to be of value for compounding statistics. Some of these are so lacking in important parts of their histories and the details of the operation and after-treatment that they convey little to the mind of the reader, excepting that "miracles" have been performed in a certain part of Italy by one Bottini.

In reading over Bottini's 23 wonderful reports here commented on we notice: 1 , that no deaths have been reported; 2 , that twenty of these patients, who had been suffering for from eight months to six years from prostatic trouble, were cured in from eleven days to three months, and all but one of them, i. e., 19, in less than one month; 3 , that these cures have been accomplished by a single cut, $2 \mathrm{~cm}$. or more in length, either in the posterior or one lateral lobe, or often in two lobes, sometimes, though rarely, in three; 4 , that the time'for the operation varied from forty-five to ninety-five seconds. The three remaining cases were very much improved.

Any investigator devising an instrument like the Bottini incisor should naturally be proud of his achievement, but it is difficult for a reader in a distant land. accustomed to observing enlarged prostates and the sequelæ of their observation, to arouse his enthusiasm to such a point as to be able to agree with the discoverer of the galvanocaustic incisor.

A reader of these reports can easily see how the heated iridioplatinum blade can glide through the prostatic bar burning a furrow through it, and reducing the height of the dam, and he can also understand how by severing and searing the ressels, the blood-supply can be sufficiently shut off to cause additional atrophy. He cannot understand, however, how a chronic cystitis which has existed for months and years can in a few days be perfectly cured, or how tone can be restored to the wall of an atonic bladder in that time.

Bottini, in some of his reports, spoke of the cases having had gonorrhea, as if he considered it a possible causative factor of the hypertrophy. I do not think that such is the case, and, in reviewing in his mind the patients whom he has treated for this trouble, he can not remember a single one where he could establish a direct relation between the two. As the patients in whom reference to such a relation was made were about forty years of age, or under, it is possible that the symptoms mentioned may have been due to some other prostatic or seminal vesicle trouble rather than to senile hypertrophy.

In speaking of his operations, Bottini says very little about the anesthetic used. He occasionally speaks of having given chloroform or cocain, but often makes no allusion to it.

The frequency with which he has resorted to permanent catheterization in after-treatment, sometimes for fiftcen days at a time, is a noticeable feature of these reports, and leads one to think that perhaps the bladder regains more tone than is gencrally supposed by being allowed to contract gradually.

It is gratifying to remark that no cases of syncope, or bladder or renal congestion followed by uremia developed in these cases, as so frequently occurs in this country.

Another thing that strikes one as most remarkable in reading over Bottini's reports is that in only one case a marked rise of temperature, that is to 104 , was mentioned. This was said to be due to malaria. The writer is, however, of the opinion that most such rises of temperature occurring after this operation in cases associated with a bad cystitis are due to septic absorption.

It was interesting to read the report of one of his cases in which a calculus was present in connection with middle lobe hypertrophy. The stone was first crushed, and then an incision made posteriorly with the incisor. it occurs to the writer that it might have been better to first perform the Bottini operation, and later the litholapaxy, as this would allow the operator to insert a larger evacuator, which is so important in litholapaxy.

Meyer has reported twelve cases. the most interesting and instructive that have been described, on account of the candor displayed in their narration, and the skillful way in which he met every obstacle.

In most of the cases he performed but one operation, although in 2 of them he was obliged to operate twice. The results were: 2 cured, 6 improved and four deaths. In most of the cases he made three incisions, and in some 
four, all of good length. In 4 he reports a marked elevation of temperature after the operation. In the first a chill was followed by temperature of 103.8 , with delirium; in the second, 103.6 , orchitis and abscess of testes; in a third, 104; in a fourth, 103. Two of these cases died. One of the cases was interesting on ac. count of the number of operations performed to no avail. They were: 1 , vasectomy, no benefit; 2 , perineal prostatectomy, no benefit; 3 , suprapubic cystotomy for inspection; 4, double castration; 5 , Bottini, under ether. The paticnt finally died. In two of his cases Meyer found it necessary to perform a suprapubic cystotomy. Most of his operations were under local anesthesia. although two were put under a general anesthetic, in one case ether, in another Schleich's.

Freudenburg has performed this operation about thirty times, but only six of his cases are here commented upon. He has done more than any other surgeon, excepting Bottini, to perfect this operation, as he has modified the instrument in a way to make it more generally acceptable to the operating surgeon. He is a careful and conscientious man and a good observer. His results, however, while they correspond with those of the other German and American surgeons, cannot compare with Bottini's, operated on by means of his oricinal crude instrument. This would lead us to think that if Bottini's reports can be accredited, the Freud(enburg modification is a failure, but such does not scem to be the opinion of other surgeons, almost all of whom have endorsed the Freudenburg instrument as the best. His reports are intelligible, and he does not claim such perfect cures as does Bottini. He has a tendency, however, to paint the pictures of his cases before opcration in a bad light, as if in an apologetic way, to explain the results that may follow. He speaks of many of them as emaciated, run down and in bad condition, with bladder and kidney complications. $\mathrm{He}$ usually makes three incisions of fair length, operates under cucain, and precedes each operation by a cystoscopic examination. Most of his cases have improved. He had two deaths.

Lohnstein has operated 12 times, but only 6 of his cases have been sufficiently well reported to be of use. He usually makes two incisions, one posteriorly and the other through one of the lateral lobes, although he has made them through both lateral lobes, omitting the posterior and anterior incision. He operated twice in 2 of his cases. Of the 6 here reviewed, 5 were improved and one died. One patient collapsed before the operation could be completed. He reports another case that collapsed on being catheterized, illustrating the mistake of too rapidly emptying the bladder. The other (i cases were only referred to, and not reported in detail, as the results were not permanent.

Simon has reported 8 cases. His reports are very interesting to read, as his methods seem different from other operators. He usually makes one incision, a posterior one, although in some he incises a lateral lobe in addition, and in others he makes three incisions. He does not seem to look at the operation so very seriously, as he performs it on patients in bad condition, suffering from cystitis, nephritis, and arteriosclerosis, having a large amount of residual urine at the time of the operation. and he allows them to return home on the following day - with at tines as much as fifteen ounces of residual urine in their bladder-and then treats them in the dispensary. Some of his cases before operation had from twenty to fifty ounces of residual urine, which was rery much improved by a posterior incision. He cysto- scoped one of these cases two days after an operation, and saw a dark groove in the site of the incision; 3 cases were reported as cured, 2 as improved and 3 died.

Crespi reported 6 of Bottini's cases. There is nothing additional to be learned from this group. They were not difficult ones, and all seem to have been benefited; 1 case was operated on twice. The most interesting of his cases is a patient who was first operated on by Bottini's method, followed by two years of relief, He then had vasectomy performed, which reduced the hypertrophy one-half, and was followed by the disappearance of blood and pus from the urine. He was then again operated on by the Bottini method, and improved. Crespi reports 4 more cases, varying in age from 64 to 78 years, 1 of which was cured in a day, another in 3 weeks and 2 others improved.

Morton has reported five cases, all improved by the operation. In each there was still some residual urine present at the last report, and the patients still had frequency of urination at night. Morton made moderatesized incisions, and operated most consistently.

Hane reported 5 cases, ranging in age from 58 to 68 and his results were fair. In 1 case he operated twice, and in the other once.

After careful operations, frequency still existed, especially at night, though not in so marked a degree. It is interesting to notice that in the cases operated on twice the bladder still contained twelve ounces of residual urine, and he had to use the catheter.

'The report of Bruce-Clarke's 4 cases as given us by Jermoli are incomplete and unsatisfactory. The operations were evidently performed under general anesthesia, and the results were all cures in a short time, notwithstanding the fact that the patients had considerable residual urine, and had suffered from prostatism for a long period.

Cecchecorelli reported 4 cases, varying in age from 41 to 60 years: all bad cases of long standing, associated with catheter life; 3 of them were cured in from nine to seventeen days, and the 1 improved for a while and then died.

Lewis reported 2 cases. These were well described. Both were operated on twice, and improved. He used air to inflate the bladder. In speaking of one case after operation, he said that the patient could empty his bladder all but two or three drams, which amount is often found in a healthy viccus. 'This statement I take exception to, as an unobstructed, healthy bladder should be able to empty itself.

Freeman has reported 2 cases wiih excellent results. He speaks of 1 patient having considerable bladder irritability after the operation. I am glad to hear this mentioned, as personally I have found it to be the most persistent and obstinate post-operation symptom that we have to contend with.

Lennander has operated in 3 cases from 65 to 77 years of age. They were all of long standing, with symptoms of obstructive cystitis; 1 was relieved, 1 discharged with a permanent fistula, and 1 died three. months after the operation. In the first case after one incision of $3 \mathrm{~cm}$. in length, the patient did not urinate for twenty-two hours. The last 2 cases were of especial interest, the first because he was made worse rather than better by the Bottini, and was then improved by a vasectomy. The second has many points of interest: 1 , both testes were very much involved by disease ascribed to catheterization; 2 , their removal had no influcnce on the size of the prostate; 3 , calculi were found in the bladder, complicating the case; 4, a suprapubic. 
cystotomy was performed; 5 , an incision $1.75 \mathrm{~cm}$. in length was made by the incisor after this, thus enabling the opcrator to see the action of the cautery knife through the suprapubic opening; 6 , a second incision, $3 \mathrm{~cm}$. long, was made by the incisor three weeks later, followed by retention, and, finally ; $r$, a permanent fistula had to be resorted to.

The reader can only condemn the presence of the assistant's finger in the rectum during the operation, as mentioned in one of the cases. as in order to detect the presence of the instrument he has to press up the anterior wall of the rectum in the region of the prostate, which might cause the heated blade to burn through, making a ureterorectal fistula. In criticising the poor results in these cases it would seem that the incisions were too short, and too few.

Downes, Weber, Chassaigne, Kreisel and Rydygier have also reported cases, most of which were improved. Rydygier's case died four days after the operation, of urinary extravasation, and septic peritonitis, due to catching the back of his instrument in a pocket in the bladder instead of behind the prostate, and thus burning through the serous surface of the bladder. This is an accident that is difficult to foresee, and can onily be avoided by making a cystoscopic examination of the bladder some time before the operation.

Rochet has reported two cuses. One died and the other was discharged seemingly improved.

The writer has performed this operation 20 times, with 2 deaths. The first 12 were all benefited and reported in the Medical Record-of the 8 others, 2 died, a total mortality of 10 per cent; 1 died of chronic Bright's disease and would not have been operated on had not the hospital interne reported the urine negative; death a few weeks after showed the cause to be chronic interstitial nephritis, which had beeen present at the time of the operation, but not to the knowledge of the operator. The other case died of sepsis. It was due to an accident, and illustrated two important features. The writer had given this operation to the house surgeon and had placed limself behind the operator with the battery. The posterior incision was being made when the writer left his post to look at the battery, which was not working well. After rectifying this, he told the operator to proceed; but during this time the patient had pulled backward, allowing the concavity of the beak to slip over the convexity of the prostate, and the cut was accordingly made through the membranous urethra into the perineum. The result of this was retention of urine, necessitating a perineal section, which was followed by sepsis and death. The two important principles illustrated by this are: 1 , that the battery should always be placed on the patient's left, where it can be seen by the operator and easily grasped by him, if necessary, with his right hand; 2 , that if the patient pulls away from the operator, as he is liable to do under local anesthesia, the operator must not allow his arm to remain stationary, but must follow up the patient so that the instrument will always remain in the same relative position until the incision is completed.

Of the remaining 18 cases, 15 have improved, and in two of them no residual urine is now present, while in the other 13 only about one-eighth to one-quarter of the amount present previous to the operation now exists; 3 others have been too recently operated on for comment at present. What these patients seem to complain of principally after the operation is irritability of the bladder.
GENERAL CONCLUSIONS.

Castration, vasectomy and iliac ligation are of very little benefit in prostatic hypertrophy.

The choice of operative procedure is between prostatectomy and the Bottini.

In commenting on these two, the writer thinks that the former is by far the most difficult and dangerous; in fact, he believes that in the hands of a good genitourinary surgeon in equally picked cases, the mortality in prostatectomy would be found to be twice that of the Bottini, and that in the hands of the general practitioner it would be three times as great.

There are certain cases suitable for enucleation and others only for the Bottini.

'Those for enucleation are the large, so-called glandular hypertrophies, where the prostate feels like a goodsized tumor by the rectum, and the urethra is felt to be much elongated and distorted by the hypertrophy. Here the operator can find something to enucleate and the pieces come away easily. The writer has performed the Bottini operation in a few of such cases on account of the refusal of the patient to undergo an enucleation and the benefit resulting from it has been surprising to him.

The cases calling for the Bottini are more those where the hypertrophy is not marked, but where rectal examination shows a marked induration, and urethral examination shows middle lobe impediment. In many of these cases an enucleation would be impossible, while in no case of the large tumor variety would a Bottini operation fail to be of benefit.

In comparing the mortality of prostatectomy with the Bottini in the future, statistics may show it in the latter to be as great as in the former, the same as is noticed in comparing prostatectomy with castration.

One of the reasons for this will probably be because both castration and the Bottini are considered easy operations, and ones that can be performed in almost any case, whereas the prostatectomy would be reserved for especially chosen and suitable cases.

Bottini is in a measure responsible for looking so lightly at the danger of this operation, as his reports resemble those of cases touched by a fairy's wand. Willy Meyer, in one of his articles, said: "It is almost incredible that this, as it seems, splendid operation should have been practiced for twenty-two years by virtually only one gentleman-namely, the inventor of the method, Enrico Bottini of Pavia-and that, in spite of the fact, he has repeatedly drawn the attention of the profession to his work."

It seems to the writer that the surgeons reading Bottini's report would consider them too good to be true, and for this reason have not paid sufficient attention to them. There appears to be among the other Italian observers engaged in this work a tendency "to follow the leader," and to paint the reports of their cases in the same rosy hue. The German and the American observers, however, go into this subject in a more thorough and scientific way. They describe their operations more clearly and do not hesitate to mention their failures and deaths. The standard of what a cure is after an operation seems to vary; some place all under this class who do not die, while others consider the same as simply improved.

It would seem from reading over these reports that the Italians must have a better tolerance for the operaations than the Germans and Americans. Such is not the case, however. The writer has a surgical service in the largest Italian hospital in this country, and 25 
per cent. of the cases operated on have been Italians, notwithstanding which he has observed less improvement in them than in others.

The class of cases demanding this operation comprises those in whom there is nearly a complete retention of urine and the patient has to depend almost entirely on the catheter, also those with the tenesmus and irritability of the bladder so great that catheterization and washing out of the bladder are not able to relieve it; again, when the cystitis is very marked or troublesome, and when hematuria is a frequent symptom. An increasing amount of residual urine is another urgent cause, or when catheterization is followed by hemorrhages or bad attacks of cystitis. The cases in which it is contraindicated are where the kidneys are badly diseased, medically or surgically, or where arteriosclerosis is marked and where the heart action is weakened through valvular or fatty changes.

The question of anesthesia is an important one. The writer does not think that either cocain or eucain relaxes spasm sufficiently, and, besides this, he is sure that most patients suffer considerably when operated on under their influence. Cocain is also said to be dangerous, and eucain in one case was found to have produced syncope. Of the general anesthetics, ether is bad for old men whose kidneys are at fault, and chloroform is bad for the heart. This has led the writer to give nitrous oxid gas in a number of his cases. The patient is put under quickly and comes out almost immediately. 'The danger in the hands of a man who understands its administration is nil.

In Colton's Dental Bureau, in Cooper Union, N. Y., it has been given over two thousand times without an accident. The patients have at times been kept under its influence for over two hours. It can be given on a full stomach.

In the ordinary operation three cuts are usually made. The first one is always posterior, the two others are on either side in cases of lateral lobe hypertrophy. In cases where the enlargement is irregular and situated principally in the middle lobe, the second incision should be anterior and the third through the larger of the lateral lobes. The posterior incision is usually 3 $\mathrm{cm}$., the anterior $2 \mathrm{~cm}$. long and the lateral $2.5 \mathrm{~cm}$. The time for each cut is usually eighty or ninety seconds, and the strength of the current from forty-five to fifty amperes.

Immediately after the operation the patient may be allowed to pass water, if he desires, and he should be put to bed. Patients are generally able to walk from the table to their beds, although it is safer to carry them.

The internal treatment consists in urinary antiseptics, diluents; and antispasmodics, if necessary. As a diluent, water taken in large quantities is usually suffcient. If it is found, however, that the patient will not dirink much, and that the amount of urine passed is below normal, its flow should be further stimulated by a mild duretic, and for this purpose I am in the habit of giving a mixture of acetate of potassium, sweet spirits of nitre, three times a day in a glass of water. As a urinary antiseptic, I generally give salol in tengrain doses three times a day, or urotropin in the same strength, the latter preferably when the urine is foul and ammoniacal. Benzoate of sodium and benzoic acid in fifteen-grain doses are also of service.

The antispasmodics are codein, morphin and belladona. Those are given for frequency, pain, tenesmus or burning. 'They may be prescribed singly or com- bined, and afford the patient a great deal of relief. Codein may be given alone or in combination with belladonna, and perhaps benzoate of sodium. Morphin is rarely used, and then only for pain. It is very efficacious in combination with the extract of belladonna, a quarter of a grain of each, in suppositories at night, in cases where there is considerable frequency, pain, burning, and tenesmus. The diet should be liquid for the first few days, then semisolid-soft-and full diet at the end of the week, if the patient has no rise of temperature and is feeling well.

If retention of urine occurs, as it frequently does, a cathether should be passed into the bladder and allowed to remain for twenty-four to forty-eight hours. On withdrawing the catheter, if the patient is still unable to pass much urine, he should be catheterized regularly until the sloughs have been passed, when, if he is still unable to urinate, a second operation should be performed. If the patient has complete retention and nothing can be passed into his bladder, he should either be aspirated suprapubically, or a perineal section performed.

If there is much hemorrhage, it can usually be stopped by a hot irrigation, and if not, a perineal section should be performed, after which a thick-walled perineal tube can be inserted into the bladder, around which gauze can be packed, thus making pressure between the sides of the tube and the cut posterior urethra.

The bowels should be moved by salines on the second day, after which they should be kept open for some days.

An elevation of temperature usually takes place on the night after the operation or on the following day, rising from 100 to $105 \cdot \mathrm{F}$. This rise generally goes down to normal after the bowels have been moved, but I have seen cases with a temperature of from 99 to 102 for some weeks after the operation. In such a case the fever usually disappears after the sloughs have been thrown off.

Where the bladder and the kidneys are very much involved a continuous temperature may indicate a disease of the latter organs, which should then be treated accordingly. Extravasion of urine and perineal abscess may occur, but it is improbable, as the tissues are practically seared and sealed by the burning process.

\section{ESTIMATION OF HEMOGLOBIN.* \\ a COMPARISON OF THE VARIOUS METHODS. BY B. M. LINNELL, M.D. \\ chICAGO.}

Most of the effort heretofore to arrive at an estimation of hemoglobin in blood has been made by means of the various color tests. 'These tests have multiplied until the catalogue of the various instruments resembles the list of "sure cure" remedies for the hives. This fact alone shows a dissatisfaction on the part of the clinician with the results obtained by the various color methods. The two instruments with which we are most familiar in this country are Von Fleischel's hemometer (the German), and Gower's (the English instrument.) The use of these instruments has demonstrated the sources of error as enumerated.

1. In color tests in general :

a. 'Two eyes rarely agree in accurate estimation of varying shades of color, thus producing individual variations.

*Presented to the Section on Practice of Medieine, at the Fiftieth Annual Meeting of the American Medical Association, held in Columbus, Ohio, June 6-9, 1899 . 